\title{
Are Damaged Lungs Protected from COVID-19? An Interesting Observation
}

\author{
Yashpal Rana ${ }^{1}$ Pudhiavan Arunachalam² \\ ${ }^{1}$ Department of Radiology and Imaging, U. N. Mehta Institute of \\ Cardiology and Research Centre, Ahmedabad, Gujarat, India \\ 2 Department of Radiology and Imaging sciences, Kovai Medical \\ Centre and Hospitals, Coimbatore, Tamil Nadu, India \\ 3 Department of Radiology, Ruby Hall Clinic and Grant medical \\ foundation, Pune, Maharashtra, India \\ ${ }^{4}$ Department of Radiology, Narayana Hrudayalaya Hospitals, \\ Bangalore, Karnataka, India \\ Indian J Radiol Imaging 2021;31:791-794.
}

Address for correspondence Yashpal Rana, MD, Department of Radiology and Imaging, U. N. Mehta Institute of Cardiology \& Research Centre, Ahmedabad 380016, Gujarat, India (e-mail: yashpalmy2@gmail.com).
The ongoing global pandemic caused by a severe acute respiratory syndrome coronavirus 2 (SARS-CoV-2) results in a respiratory tract infectious disease named the novel coronavirus disease 2019 (COVID-19). ${ }^{1}$ The disease has exponentially spread across the world, since the first reported case in Wuhan in December 2019. ${ }^{2}$ India is one of the worst-hit countries and is currently recovering from the second wave of the disease.

High-resolution computed tomography (HRCT) scanning of the chest can be a useful modality in assessing the degree of lung involvement and various complications of COVID-19. Radiological patterns of lung involvement on HRCT varies widely in the disease course., ${ }^{3,4}$ The typical imaging features of early COVID-19 are well described and include patchy, rounded, peripheral segmental, or subsegmental groundglass opacities (GGOs), with or without consolidation., ${ }^{5,6}$ Evidence is also emerging to show that nearly one-third of patients with moderate-to-severe COVID-19 may develop pulmonary fibrosis in intermediate-term follow-up., ${ }^{7,8}$

Patients with known comorbidities are shown to have poor outcomes with COVID-19. ${ }^{7,9}$ One would imagine that patients who have lung damage secondary to prior infections, such as tuberculosis (TB) or emphysema, may have worse lung involvement with COVID-19 and take a longer time for recovery. Over the last few months, we have found evidence that contradicts the above supposition. In various imaging discussions on COVID-19, interesting multicentric observations are drawn which depict that the lung parenchyma which is previously affected by lung pathologies, such as TB, cystic bronchiectasis/fibrosis, emphysema, and others, show relative sparing or lesser degree of involvement of COVID-19.

We present this very interesting case where an aspirated tablet was lodged in the bronchus intermedius. The patient had extensive involvement of the entire left lung and right upper lobe, while the right middle and lower lobes were nearly spared with very few ground-glass infiltrates (-Fig. 1). Similar findings were also seen in another case of an adult male with known left lung bronchiectasis and active COVID-19. Here, we saw that most of the left lung is spared, while there are diffuse infiltrates in the right lung (-Fig. 2). We have made similar observations in many patients who have old TB-related fibrosis. In the presented case 3 , it is seen that the fibrotic lung parenchyma seems to be either completely spared or only partially involved with COVID-19 ( - Fig. 3). In - Fig. 4, we can see two patients with active COVID-19 and a past history of TB showing sparing of unilateral upper lobes from active GGOs. In both these cases, the upper lobe fibrosis seems to have some protective effect and hence not involved with active infiltrates.

Two popular pathophysiologies of SARS-CoV-2 focus on angiotensin-converting enzyme 2 (ACE 2) receptors and endothelial damage by the virus. In the former, viral spike proteins are thought to bind to ACE 2 receptors for attachment to host cells. The ensuing proinflammatory cytokinemediated immune overreaction is the major cause of lung damage and acute respiratory distress syndrome (ARDS) in severe cases. The expression level of ACE 2 may differ
DOI https://doi.org/ $10.1055 / \mathrm{s}-0041-1735917$. ISSN 0971-3026. (c) 2021. Indian Radiological Association. All rights reserved. This is an open access article published by Thieme under the terms of the Creative Commons Attribution-NonDerivative-NonCommercial-License, permitting copying and reproduction so long as the original work is given appropriate credit. Contents may not be used for commercial purposes, or adapted, remixed, transformed or built upon. (https://creativecommons.org/ licenses/by-nc-nd/4.0/)

Thieme Medical and Scientific Publishers Pvt. Ltd., A-12, 2nd Floor, Sector 2, Noida-201301 UP, India 


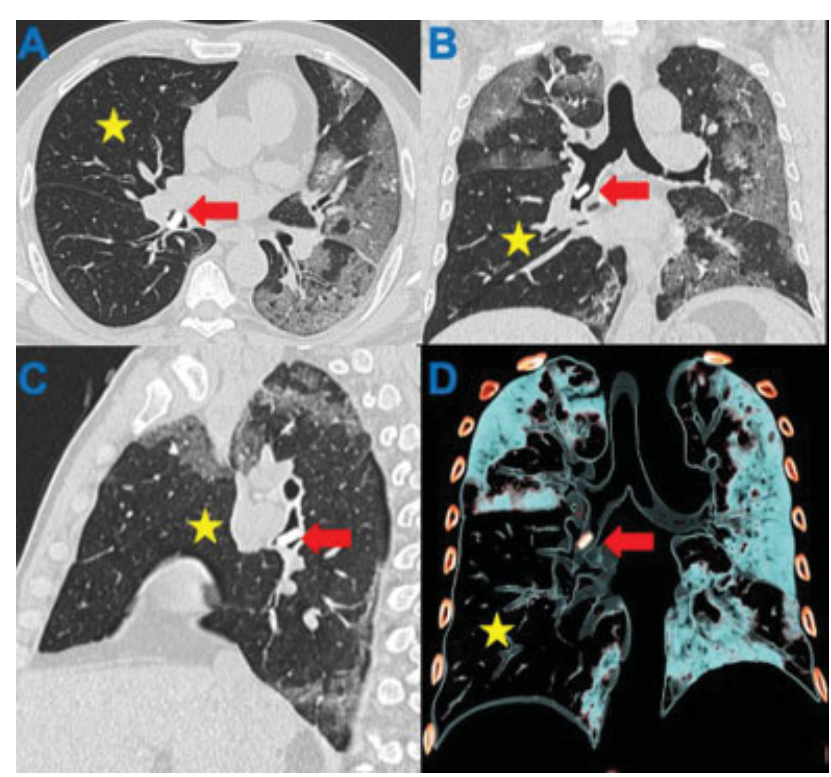

Fig. 1 (A) Axial, (B) coronal, (C) sagittal MPR (multiplanar reconstruction) and (D) coronal VRT (volume-rendered technique) thin HRCT images of an adult male patient. A radiodense round foreign body (consistent with tablet) is shown in the bronchus intermedius (red arrow). Note, relative sparing of right middle and lower lobar segments (yellow star) by COVID-19 disease while extensive involvement of rest of lung segments on either side. COVID-19, novel coronavirus disease; HRCT, high-resolution computed tomography.
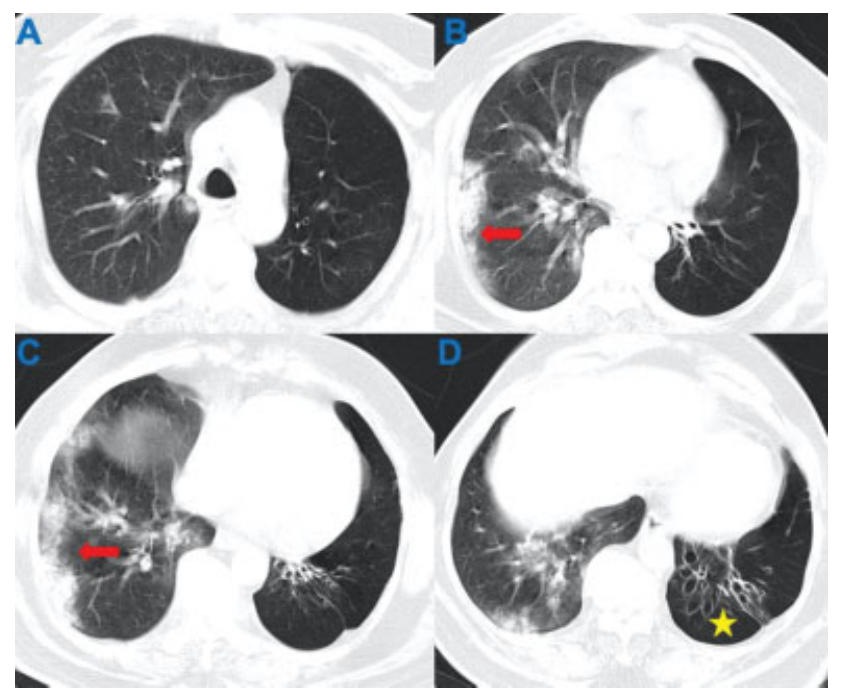

Fig. 2 (A-D) Axial images of HRCT chest study at different levels. Peripheral ground glass changes are seen in the right lung in keeping with proven COVID-19 (red arrow). Widespread air trapping is seen in the left lung with overt cylindrical bronchiectasis in left lower lobe (yellow star). Notice the near complete sparing of the left lung as part as COVID-19 changes are concerned. COVID-19, novel coronavirus disease; HRCT, high-resolution computed tomography.

between patients accounting for differences in severity of clinical disease..$^{10}$ In the other theory, COVID-19 is postulated to be a predominantly vascular disease. The virus leads to endothelial injury with increased fibrinogen and related products which in turn lead to macro- and microvascular

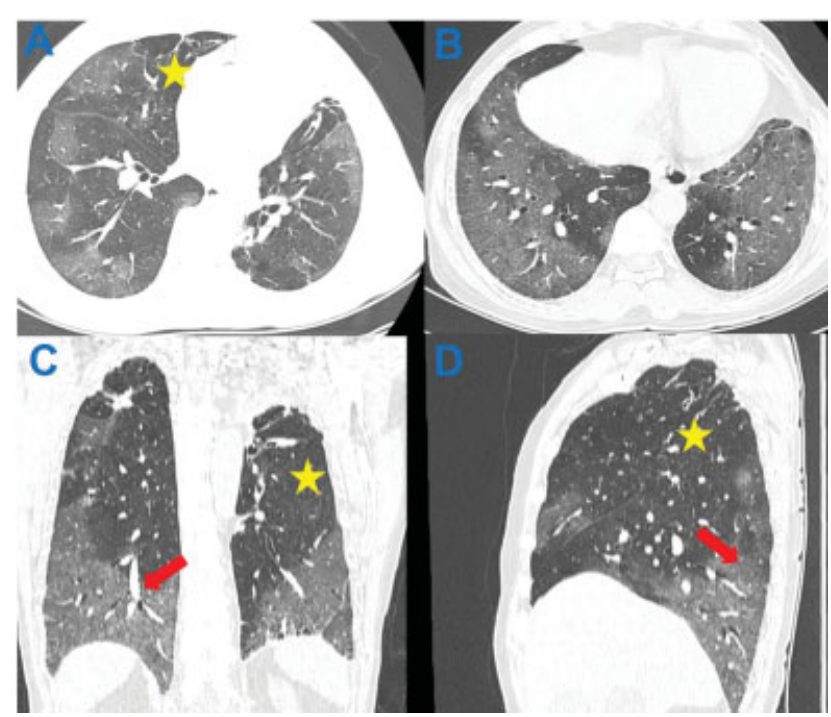

Fig. 3 (A, B) Axial, (C) coronal and (D) sagittal MPR (multiplanar reconstruction) images of an adult male patient with proven COVID-19. Widespread areas of ground glass opacities are seen in both lungs (red arrow). Within these areas you can see partial sparing of the medial segment of the right middle lobe (yellow star in A), left upper lobe/lingula (yellow star in C), and the right upper lobe (yellow star in D) with underlying fibrotic changes in the lungs. Traction bronchiectasis/reticulation is seen in these segments in keeping with prior history of TB. COVID-19, novel coronavirus disease; TB, tuberculosis.

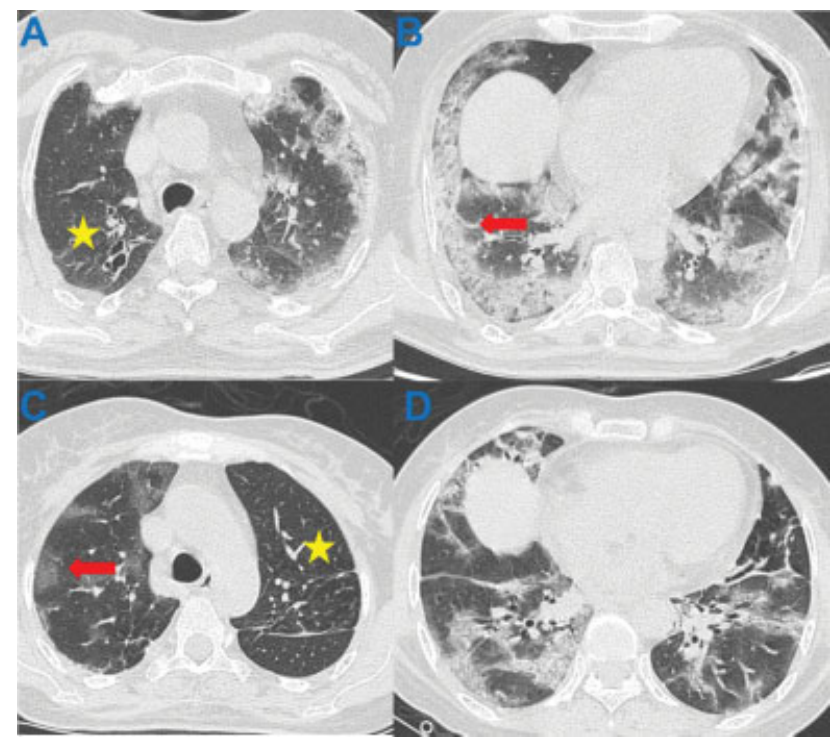

Fig. 4 (A, B) Axial images of one patient and (C, D) axial images of another patient with COVID-19. Extensive areas of lung involvement with active infection is seen in both patients (red arrow). At the same time we can see areas of right upper lobe (A) and left upper lobe (B) that demonstrate fibrotic changes (yellow star) are spared from any ground glass opacities or active inflammation. COVID-19, novel coronavirus disease.

thrombotic disease in these patients. ${ }^{11,12}$ Multiple studies have shown a correlation between clinical severity and raised fibrinolytic substances, such as D-dimer and the von Willebrand factor, supporting this concept. ${ }^{13,14}$ 
All the cases presented here have a common finding, that is, the lung segments with bronchiectasis/fibrosis seem to be spared or less affected compared with the other segments. The most likely explanation for this is related to reduced ventilation (V) and/or perfusion (Q) of these segments compared with the normal lung segments. Ventilation/ perfusion $(\mathrm{V} / \mathrm{Q})$ mismatch is well known in patients with bronchiectasis, cystic fibrosis, interstitial lung diseases, and emphysema. ${ }^{15}$ With reduced ventilation, the body autoregulates by hypoxic pulmonary vasoconstriction leading to a reduction in perfusion to the involved segments. This reduced perfusion appears to be providing some protection to these lung segments from COVID-19. Another possibility is alteration at cell membrane attachment sites in these fibrotic segments. A similar observation has also been made in one report which looked at cystic fibrosis and COVID-19. ${ }^{16}$

These observations can have a significant impact on our understanding of the disease and its pathophysiology. We encourage our colleagues and other researchers to further look into this concept, perhaps this may open new avenues for novel preventive and/or therapeutic strategies.

\section{Ethical Consideration}

No patient identifiable data have been used.

\section{Conflict of Interest}

The authors have no conflict of interests related to this work.

\section{References}

1 World Health Organisation. Report of the WHO-China joint mission on coronavirus disease 2019 (COVID-19). Accessed August 17, 2021 at: https://www.who.int/docs/default-source/coronaviruse/whochina-joint-mission-on-covid-19-final-report.pdf

2 Guan WJ, Ni ZY, Hu Y, et al; China Medical Treatment Expert Group for Covid-19. Clinical characteristics of coronavirus disease 2019 in China. N Engl J Med 2020;382(18):1708-1720
3 Shi H, Han X, Jiang N, et al. Radiological findings from 81 patients with COVID-19 pneumonia in Wuhan, China: a descriptive study. Lancet Infect Dis 2020;20(04):425-434

4 Chung M, Bernheim A, Mei X, et al. CT imaging features of 2019 novel coronavirus (2019-nCoV). Radiology 2020;295(01): 202-207

$5 \mathrm{Ng}$ M-Y, Lee EYP, Yang J, et al. Imaging profile of the COVID-19 infection: radiologic findings and literature review. Radiol Cardiothorac Imaging 2020;2(01):e200034

6 Raj V, Jagdish J. Computed tomography (CT) chest imaging in diagnosis and management of patients with COVID-19. J Indian Med Assoc 2020;118(06):35-42

7 Han X, Fan Y, Alwalid O, et al. Six-month follow-up chest CT findings after severe COVID-19 pneumonia. Radiology 2021;299 (01):E177-E186

8 Urciuoli L, Guerriero E. Chest CT findings after 4 months from the onset of COVID-19 pneumonia: a case series. Diagnostics (Basel) 2020;10(11):899

9 Sanyaolu A, Okorie C, Marinkovic A, et al. Comorbidity and its impact on patients with COVID-19. SN Compr Clin Med 2020;2 (08): $1-8$

10 Yuki K, Fujiogi M, Koutsogiannaki S. COVID-19 pathophysiology: a review. Clin Immunol 2020;215:108427

11 Siddiqi HK, Libby P, Ridker PM. COVID-19 - a vascular disease. Trends Cardiovasc Med 2021;31(01):1-5

12 Colling ME, Kanthi Y. COVID-19-associated coagulopathy: an exploration of mechanisms. Vasc Med 2020;25(05):471-478

13 Goshua G, Pine AB, Meizlish ML, et al. Endotheliopathy in COVID-19-associated coagulopathy: evidence from a single-centre, cross-sectional study. Lancet Haematol 2020;7(08): e575-e582

14 Helms J, Tacquard C, Severac F, et al; CRICS TRIGGERSEP Group (Clinical Research in Intensive Care and Sepsis Trial Group for Global Evaluation and Research in Sepsis) High risk of thrombosis in patients with severe SARS-CoV-2 infection: a multicenter prospective cohort study. Intensive Care Med 2020;46(06): 1089-1098

15 Sarkar M, Niranjan N, Banyal PK. Mechanisms of hypoxemia. Lung India 2017;34(01):47-60

16 Bezzerri V, Lucca F, Volpi S, Cipolli M. Does cystic fibrosis constitute an advantage in COVID-19 infection? Ital J Pediatr 2020;46 (01):143 